\title{
Téoros
}

Revue de recherche en tourisme

\section{Du musée-conservateur au musée-entrepreneur}

\section{Elsa Vivant}

Volume 27, numéro 3, automne 2008

Nouveaux Musées, Nouveaux Tourismes

URI : https://id.erudit.org/iderudit/1070783ar

DOI : https://doi.org/10.7202/1070783ar

Aller au sommaire du numéro

Éditeur(s)

Université du Québec à Montréal

\section{ISSN}

0712-8657 (imprimé)

1923-2705 (numérique)

Découvrir la revue

\section{Citer cet article}

Vivant, E. (2008). Du musée-conservateur au musée-entrepreneur. Téoros, 27(3), 43-52. https://doi.org/10.7202/1070783ar d'utilisation que vous pouvez consulter en ligne.

https://apropos.erudit.org/fr/usagers/politique-dutilisation/ 


\section{Du musée-conservateur au musée-entrepreneur}

\section{Elsa Vivant}

La création des premiers musées visait à ouvrir les portes des palais et à offrir au regard du peuple le plaisir du spectacle des trésors et des œuvres d'art qu'ils recelaient. Aujourd'hui, les fonctions originelles des musées demeurent le cœur de leur activité et leur raison d'être : préserver, étudier, éduquer et exposer une collection. Pour mettre en œuvre ces missions, ils font face à de nouveaux enjeux tels que la gestion des flux de visiteurs et la recherche de financement. La médiatisation des expositions, les rénovations visant à agrandir les musées et la mise en œuvre d'une logique de rentabilité sont révélateurs de l'intégration des musées à l'industrie de la consommation culturelle et de l'imbrication croissante entre culture et société de marché. En effet, les évolutions des musées doivent être considérées dans un contexte plus large où l'augmentation du temps des loisirs et l'accroissement des mobilités accompagnent la commercialisation de la culture et la massification du tourisme. Même si les musées sont des institutions à but non lucratif, leur activité s'inscrit aujourd'hui dans une logique économique capitaliste, au cœur d'une société de marché où tout peut être acheté, y compris les biens culturels, et où les critères quantitatifs d'évaluation (fréquentation, ressources de billetterie, etc.) prennent le pas sur le qualitatif (Martel, 2006). Ainsi, les musées, de plus en plus nombreux, sont en compétition entre eux pour attirer les visiteurs, mais aussi avec les autres lieux de culture et de divertissement. Publics ou privés, populaires ou élitistes, petits ou grands, les lieux de consommation culturelle se multiplient dans l'espace urbain. Cinémas multiplexes, musées, espaces d'expositions, galeries de jeux, salles de spectacles, mais aussi casinos, restaurants, discothèques, cafés, parcs d'attractions... les citadins et les visiteurs sont submergés par l'offre de loisirs. Dans ce contexte concurrentiel, les musées s'ingénient à proposer une offre à la fois exigeante et accessible, savante et ludique. Ils sont aujourd'hui porteurs d'enjeux et d'attentes qui dépassent l'atmosphère feutrée des galeries. Leurs impacts (supposés ou espérés) sur l'économie locale (notamment sur l'industrie touristique) amènent les décideurs locaux à les considérer comme des attractions au service du développement économique du territoire ${ }^{1}$, autant, si ce n'est plus, que comme des espaces d'éducation culturelle, de délectation et de plaisir artistique. D'ailleurs, un récent rapport gouvernemental britannique tend à mettre en avant l'impact économique des musées plus que leurs fonctions d'éducation, de conservation et de recherche ${ }^{2}$. L'affichage d'une nouvelle hiérarchisation des objectifs des musées renforce une critique formulée par de nombreux observateurs d'un risque de fourvoiement des musées vers des dérives mercantiles, oubliant leur vocation première (Clair, 2007 ; Mathur, 2005 ; Van Aalst et Boogaarts, 2002; Werner, 2005; Wu, 2002).

Si les rôles, les responsabilités et les activités des institutions muséales visent une démocratisation de l'accès à la culture, les modalités de cet engagement s'inscrivent aujourd'hui dans le contexte socio-historique de l'avènement du néolibéralisme global. Pour le décrire, le géographe marxiste David Harvey (1989) a analysé le tournant entrepreneurial qui a affecté les politiques urbaines dans les années 1980, marquées par la décentralisation politique, le désengagement des États et la mise en concurrence des territoires. Les villes ne sont plus uniquement des fournisseuses de services pour leurs habitants (logique managériale), mais des entrepreneuses qui prennent des risques pour conquérir de nouveaux marchés et attirer des investisseurs, des entreprises, des habitants et des touristes. Ces villes-entrepreneuses mettent en place de nouveaux partenariats, avec des acteurs publics et privés, à partir desquels s'organise le partage des risques stratégiques. De manière similaire, ce tournant entrepreneurial affecte les modes d'administration des musées. En effet, un parallèle peut être établi entre la compétition interurbaine pour l'attraction des investisseurs, des touristes et des visiteurs, et celle entre les (grands) musées qui se battent pour augmenter leur fréquentation et renforcer leur réputation. Ainsi, au-delà de leurs missions de conservation et d'éducation, l'imbrication de différentes logiques de gestion a permis la mise en place depuis une vingtaine d'années d'une logique entrepreneuriale dans l'administration des musées. Ce basculement sera analysé ici par la mise en perspective de différentes évolutions : la rationalisation de la gestion des collections; la mise en œuvre de nouveaux partenariats entre institutions muséales et avec le secteur privé à l'occasion de grandes expositions; le choix de mode de gestion inspiré du monde de l'entreprise; le rôle croissant des acteurs privés dans le financement et l'administration, notamment par l'implication des mécènes; le triomphe de l'architecture iconique et la réorganisation des espaces; la prise de 
risque dans les choix stratégiques de développement ; la mise en œuvre de logique de marque muséale, voire la création de branches de musées.

\section{L'exposition : support de nouveaux partenariats}

Les grandes expositions régulièrement organisées par les musées sont le prétexte et le support de la mise en place de nouveaux partenariats, à la fois avec d'autres institutions muséales et avec des acteurs privés soutenant financièrement ces opérations $^{3}$. De grandes expositions, organisées conjointement par plusieurs musées de différents pays, circulent entre eux, à travers le monde ${ }^{4}$. La coopération entre les institutions pour l'organisation des expositions à l'échelle internationale permet de rassembler des œuvres provenant de collections différentes, sous la direction d'un commissaire d'exposition. Ces expositions offrent au public un événement unique, ayant une orientation conceptuelle ou artistique forte, selon les visions des commissaires qui revendiquent de plus en plus la reconnaissance et la valorisation de leurs compétences en tant que créateurs (Moureau et Sagot-Duvauroux, 2001). Qualifiées de blockbusters par analogie aux superproductions hollywoodiennes, elles augmentent l'attractivité des musées et sont des motifs de visites. Beaucoup les critiquent et les considèrent comme le symbole du tournant mercantile des institutions muséales. Par souci d'efficacité (en terme de fréquentation), ce sont souvent les œuvres les plus connues, les artistes les plus célèbres, les courants les plus populaires qui sont représentés, laissant peu de place à la nouveauté, à l'initiation et à la découverte de talents nouveaux ou méconnus (Lai, 2004 ; Rapetti, 2001$)^{5}$, malgré quelques notables exceptions comme l'exposition Mélancolie, génie et folie en occident, conçue par Jean Clair, présentée aux Galeries nationales du Grand Palais et à la Neue Nationalgalerie de Berlin. Une exposition sur Picasso ou sur les peintres impressionnistes a toutes les chances d'attirer les foules et les sponsors $^{6}$. Si, pendant la durée du prêt, les œuvres ne sont pas visibles dans leur musée d'origine, les expositions itinérantes sont un moyen d'exposer davantage d'œuvres des collections en les faisant circuler. Malgré les coûts de transport et d'assurance élevés dus aux risques de mauvaises manipulations, de dégradation ou de vol durant les transferts, organiser une exposition à plusieurs permet de réunir un plus large choix d'œuvres, de partager les coûts, de faire des économies d'échelle (notamment sur les produits dérivés) et de renforcer mutuellement les réputations des différentes institutions partenaires. Préparer une exposition avec une institution très connue est certes un mode de légitimation de la qualité et de la pertinence des choix des commissaires d'exposition. Les expositions internationales les plus visitées sont le plus souvent organisées par quelques grands musées, renforçant la logique de starification des institutions culturelles (Lai, 2004). Un club de "musées-stars» se constitue, entre lesquels circulent les expositions, les œuvres et les visiteurs. Ce club se renforce par la signature d'accords spécifiques entre musées, par exemple le musée du Louvre et le High Museum d'Atlanta' ou le musée de l'Hermitage, qui a multiplié les partenariats (avec le Courtaud Institute de Londres, la fondation Guggenheim de Las Vegas et Nieuwe Kerk d'Amsterdam) dans le but de trouver les financements nécessaires à la rénovation du musée de Saint-Pétersbourg.

Une exposition temporaire est aussi une occasion de nouer des partenariats avec des entreprises privées qui la soutiennent financièrement en échange d'une visibilité sur l'événement (par exemple par l'apposition du logo du sponsor sur les supports de communication). Les sponsors les plus importants jouissent de certains privilèges comme la privatisation du musée pour des événements internes à l'entreprise (une assemblée d'actionnaires par exemple). Les sponsors et les partenaires privés s'associent d'autant plus à l'événement que, par leur dimension événementielle et par une stratégie de communication pointue, les expositions sont l'objet d'un traitement médiatique important ${ }^{8}$. La participation financière d'une entreprise peut parfois résulter en l'attribution du nom d'une marque à un événement, à une exposition temporaire ou à un prix, faisant presque oublier l'objet des activités de l'entreprise, dépassé par les retombées médiatiques de son engagement pour les arts. Associer son nom, son logo et son image à un événement artistique majeur participe à la construction de l'identité de l'entreprise (corporate identity), par association d'idée entre qualité esthétique et qualité de ses produits, et lui permet de renforcer sa valeur symbolique, voire de transformer son image de marque (Lindemann, 2001). Qui se souvient qu'Unilever est le leader mondial des produits ménagers avant d'être le nom de l'exposition-évènement des hivers londoniens à la Tate Modern? Le glamour associé au prix Hugo Boss d'art contemporain, dont les lauréats sont exposés $\mathrm{au}(\mathrm{x})$ musée(s) Guggenheim, se reflète-t-il sur la valeur ou la qualité esthétique d'un costume? L'engagement des entreprises dans l'organisation d'événements artistiques bouleverse le champ de la reconnaissance artistique. Ces entreprises produisent un nouveau discours sur l'art contemporain et s'auto-désignent comme arbitre des élégances et des goûts (Wu, 2002). Cette imbrication des intérêts du monde de l'art et de ceux des entreprises est particulièrement prononcée dans le cas des industries du luxe. Ainsi, le principal groupe mondial du luxe (LVMH) s'engage dans le monde de l'art contemporain par différentes portes. Certaines de ses enseignes ont commandé des créations-objets à des artistes, comme Takashi Murakami qui a dessiné un sac pour Louis Vuitton. Le groupe LVMH, ou une de ses marques, est un sponsor majeur des événements artistiques parisiens, notamment des expositions du Centre Georges Pompidou. Le président du groupe, Bernard Arnaud, est un collectionneur important dont la collection sera présentée dès 2012 dans la future galerie de la fondation d'entreprise Louis Vuitton pour la création, construite à Paris par Franck Gehry. Au tournant des années 2000, Bernard Arnaud était également propriétaire de la maison de vente aux enchères Philips (la troisième mondiale) et du bureau de commissaire-priseur Trajan. D'autres entreprises se sont impliquées de manière plus controversée dans l'organisation d'expositions. Par exemple, Hermès (fabricant de bagagerie de luxe) était l'unique sponsor d'une exposition sur les sacs au Musée des arts décoratifs à Paris; le musée Guggenheim a lui organisé 
une exposition, très critiquée, autour des créations d'Armani, après une donation de celui-ci de près de 15 millions de dollars. Le parrainage d'événements artistiques relève alors explicitement d'une stratégie de communication d'entreprise et non pas d'une action désintéressée.

\section{De la conservation des collections à la gestion des stocks d'œuvres : rationalisation ou prise de risque?}

Les contraintes techniques de la conservation des œuvres ne permettent pas leur exposition permanente, ce qui demanderait d'ailleurs des surfaces d'exposition très importantes. Or la conservation des réserves d'œuvres pose également des problèmes de gestion et engendre des coûts que certains considèrent comme trop importants. C'est du moins en termes de gestion des stocks que certains abordent en France le tabou de la vente des œuvres des collections des musées. Cette pratique reste marginale et quasi interdite pour les collections nationales françaises, mais des voix s'élèvent officiellement pour autoriser la vente de certaines œuvres des collections des musées labellisés "Musée de France». En effet, un député de la majorité conservatrice (Jean-François Mancel) a déposé le 27 septembre 2007 une proposition de loi «tendant à établir une réelle liberté de gestion des établissements culturels", dont le but est de réduire les «stocks» d'œuvres des musées dont la gestion et les coûts seraient "étouffants [sic] " et de dégager des ressources financières par la commercialisation et la valorisation des surplus d'œuvres ${ }^{9}$. Cette proposition s'inscrit dans un débat ouvert par la Commission sur l'économie de l'immatériel qui recommandait en 2006 de donner la possibilité aux musées français de «louer et de vendre certaines de leurs œuvres» et d' «avoir une gestion dynamique de leurs collections» afin de "renforcer [leur] rayonnement " (Lévy et Jouyet, 2006 : 123 ${ }^{10}$. Depuis, le président Nicolas Sarkozy a explicitement sommé la ministre de la Culture et de la Communication, Christine Albanel (2008), dans la lettre de mission qu'il lui a adressée le $1^{\text {er }}$ aout 2007 , d'«engag[er] une réflexion sur la possibilité pour les opérateurs publics d'aliéner des œuvres de leurs collections, sans compromettre naturellement le patrimoine de la Nation, mais au contraire dans le souci de le valoriser au mieux ${ }^{11}$. Jacques Rigaud, chargé de mener cette réflexion, modère dans son rapport les velléités de ceux qui rechercheraient des trésors cachés dans les réserves des grands musées en rappelant que, dans l'ensemble, ces œuvres ne sont pas toutes en bon état ou d'un intérêt spécial et en affirmant que, au Louvre ou au musée d'Orsay, «tout ce qui est montrable est montré» (2008: 12). II retrace les enjeux scientifiques et artistiques de telles réserves et rappelle que la loi relative au label des «Musées de France » de 2002 instaure juridiquement l'inaliénabilité des collections tout en instaurant, au cas par cas, la possibilité de déclasser les œuvres des collections publiques (possibilité certes jamais mise en œuvre jusqu'alors). II en conclut qu'il conviendrait de s'en tenir aux possibilités offertes par la loi de 2002 de ne pas étendre «la portée de l'exception d'aliénabilité des collections publiques au-delà d'une mise en œuvre sincère et expérimentale du déclassement », tout en rappelant que la loi permet la «valorisation" des collections de multiples manières (Id. : 35). En fait, le législateur français s'est inspiré d'une pratique ayant cours ailleurs, notamment aux États-Unis : le deaccessioning. Ce terme signifie (et masque) la pratique de vente de certaines $œ u v r e s$ des collections de musées ${ }^{12}$. Les œuvres jugées mineures ou redondantes dans la collection peuvent être vendues à la condition que les fruits de la vente soient réinvestis dans de nouvelles acquisitions. Pour l'heure, il n'est pas accepté (moralement) que ces reventes servent à alimenter le budget de fonctionnement du musée ou que les revenus qui en découlent soient investis dans de nouvelles réalisations immobilières.

Les critiques déplorent que cette pratique vide les collections des grands musées dont les œuvres iraient enrichir des collections privées. Ils rappellent également que les œuvres qui sont aujourd'hui considérés comme mineures, et dont les musées pourraient se dessaisir, seront peut-être demain des chefs-d'œuvre d'une valeur inestimable. Les canons de la reconnaissance artistique sont très subjectifs, contextuels et volatiles. Les modes, les reconnaissances subites et les oublis rapides ont marqué l'histoire de l'art (Wu, 2002). Enfin, le deaccessioning pose un problème d'ordre déontologique lorsque l'œuvre mise en vente est issue d'une collection privée donnée au musée. La vente serait une offense aux volontés du collectionneur, pour qui le don au musée est un gage de pérennisation de sa collection, et risquerait de décourager les futurs legs (Feldstein, 1991). D'un autre point de vue, le deaccessioning peut être considéré comme l'occasion pour de nouvelles institutions muséales d'enrichir leur collection d'œuvres de maitres jusqu'à présent inaccessibles sur les marchés de l'art car appartenant aux principaux musées du Vieux Continent et du nord-est des ÉtatsUnis. Ce «transfert» d'œuvres participerait à l'émergence d'une nouvelle géographie de l'offre muséale portée par la volonté de quelques mécènes-collectionneurs. Par exemple, la fondation de la famille Walton (propriétaire de la chaîne de supermarchés Wal-Mart) a acheté quelques chefs-d'œuvre de musées newyorkais qu'elle souhaite (ou qu'elle déclare souhaiter) exposer dans le musée qu'elle construit dans l'Arkansas (Martel, 2006). Cette rationalisation progressive et risquée de la gestion des collections reflète le mouvement plus profond de rationalisation de l'administration des musées eux-mêmes.

\section{L'influence croissante du monde de l'entreprise dans l'administration des musées}

La gestion des musées est conditionnée par un paradoxe : ils sont potentiellement très riches (par la valeur potentielle des chefs-d'œuvre qu'ils abritent), mais les coûts de maintenance des collections (restauration, stockage, exposition) et les dépenses quotidiennes (notamment les salaires) ne sont pas couverts par leurs revenus propres (Benhamou, 2004 ; Feldstein, 1991). Les musées, publics et privés, sont des organisations sans but lucratif, mais qui évoluent dans un système capitaliste, régi par des logiques de compétition et où, le plus souvent, les plus 
gros captent l'essentiel des ressources (Frey, 1998). Pour réduire leurs coûts de fonctionnement, ils mettent en œuvre des logiques de management qui s'inspirent des modes de gestion de l'entreprise.

L'usage de termes comme "box office», «audit», "évaluation» ou «contrôle de gestion» montre l'importance prise par la vision des managers sur celle des conservateurs. L'appropriation par les musées de techniques de gestion propres au monde des entreprises révèle leur entrée dans l'ère entrepreneuriale. Outre la rationalisation de la gestion des collections ellesmêmes, caricaturée par les pratiques de deaccessioning, la rationalisation de la maintenance des musées les amène à sous-traiter un certain nombre d'activités non directement liées à leur fonction (nettoyage, surveillance). Ils peuvent également louer certains espaces à des entreprises privées, par exemple dans le domaine de la restauration. Plus surprenant, certaines institutions font appel à des partenaires privés pour sous-traiter la gestion d'établissements ou pour l'organisation et la conception d'expositions temporaires. L'Institut de France ${ }^{13}$ a délégué à une entreprise privée, Culture Espaces ${ }^{14}$, la gestion et l'animation de certains de ses musées et monuments ${ }^{15}$. Le Musée du Luxembourg (dépendant du Sénat) a signé un contrat pour la période 2005-2008 avec l'entreprise Sylvestre Verger pour la conception, l'organisation et l'animation de ses expositions temporaires, après avoir lancé un appel d'offres auquel avaient répondu une compagnie canadienne, Lord, et la Réunion des musées nationaux ${ }^{16}$ (Azimi, 2003). Enfin, la rationalisation touche également l'administration des musées, notamment les musées nationaux français qui, d'une part, voient leur domaine s'élargir ${ }^{17}$ et, d'autre part, accèdent à l'autonomie administrative par le statut d'«Établissement public » induisant la mise en place de nouvelles normes comptables et de nouveaux modes de gestion (évaluation et contractualisation des moyens et des objectifs). Une logique de performance, basée sur les taux de fréquentation, la collecte de fonds privés et la visibilité des collections hors du musée, s'immisce dans ce monde feutré et a priori hors de la sphère marchande. Pour assumer les conséquences de ces évolutions, les directeurs de musée développent de nouvelles compétences de gestionnaire autant, si ce n'est plus, que de conservateur. D'ailleurs, les musées, en particulier les grands musées privés américains, recrutent de plus en plus de diplômés en gestion, non plus uniquement des historiens d'art. La gestion d'endowments, ou fonds de dotation ${ }^{18}$, de plus en plus importants, le besoin de mettre en place des stratégies de recherche de financement innovantes et l'adoption progressive d'un mode de gestion entrepreneurial incitent les musées à engager des directeurs qui ont un diplôme de type MBA obtenu d'une des universités les plus prestigieuses (et donc les plus coûteuses). Les salaires qu'ils réclament se rapprochent de ceux du privé.

Face à la hausse des coûts de fonctionnement et à la baisse des financements publics, les musées diversifient leurs sources de revenus. Pour consolider leurs ressources propres (billetterie, produits dérivés) ${ }^{19}$, les institutions sollicitent la générosité des acteurs privés (individus ou entreprises) qui, par leurs dons et legs d'œuvres, enrichissent les collections et, par leur mécénat financier, mettent en œuvre de nouvelles réalisations immobilières. L'action philanthropique ne se cantonne pas à sa dimension financière. Dans les musées privés à but non lucratif, les principaux mécènes participent aux conseils d'administration (board of trustees). Être membre du board of trustees est un mode de distinction de l'élite économique, notamment aux États-Unis où la philanthropie est un élément fondamental de l'éthos du capitalisme. La volonté de laisser une trace positive à la postérité a été à l'origine de l'action philanthropique de riches entrepreneurs au XIX ${ }^{e}$ siècle ${ }^{20}$. Aujourd'hui encore, le prestige social et le besoin d'implication dans la vie de la communauté (et quelques incitations fiscales) expliquent en grande partie le poids de la philanthropie dans l'économie américaine. Le don philanthropique est aussi un symbole de pouvoir et de domination. Lieu de socialisation de l'élite, le board of trustees exerce un pouvoir décisionnaire important quant aux choix stratégiques du musée, tant en matière de conservation que de gestion. L'implication de riches entrepreneurs dans les conseils influence progressivement les modes d'administration des musées, où sont adaptées de nombreuses recettes et modalités de la gestion des entreprises. Par leur engagement philanthropique dans l'administration des musées, les entrepreneurs instillent progressivement leur vision et leur méthode de management. Les boards of trustees fonctionnent comme des clubs très fermés que les principaux mécènes utilisent eux-mêmes comme un espace de légitimité et de prestige sociaux, autant qu'un espace de socialisation professionnelle, un lieu où une confiance s'installe entre des chefs d'entreprises qui interagissent également entre eux dans la sphère des affaires. Aujourd'hui, certains hommes d'affaires ayant fait une rapide et colossale fortune (notamment dans les nouvelles technologies) cherchent à prouver leur légitimité sociale par le biais de leur appartenance à ces boards et, pour cela, sont prêts à faire des dons très importants aux institutions culturelles. Ces nouveaux philanthropes, formés à l'école de la nouvelle économie, promeuvent une philanthropie pragmatique (venture philanthropy) qui s'appuie sur la prise de risque et l'évaluation de l'efficacité à la fois de l'action philanthropique elle-même et du retour sur investissement (en termes de niche fiscale) du mécène-investisseur (Abeles, 2002).

Mais, considérer qu'une part importante des ressources des musées doit provenir des dons de particuliers ou d'entreprises expose leur budget aux aléas économiques et aux fluctuations des modes de légitimité sociale ${ }^{21}$. L'hybridation croissante entre public et privé du financement et de la gestion des musées risque de fragiliser le budget et le fonctionnement des musées si une situation macroéconomique ralentit l'implication des mécènes (Benhamou, 2004; Schuster, 1998). Certains critiques interprètent l'importance du rôle des mécènes privés dans l'administration et les prises de décision comme une confiscation de l'action culturelle par une élite économique (Martel, 2006). Cette participation des mécènes dans l'administration d'un musée peut engendrer des conflits d'intérêt lorsque ceux-ci sont également 
collectionneurs. Chin-Tao Wu met ainsi en évidence que des collectionneurs peuvent influencer la politique artistique (de conservation, d'acquisition et d'exposition) des musées dont ils sont administrateurs : en prêtant des œuvres de leur collection, ils mettent en valeur l'œuvre et l'ensemble de leur collection, comme cela fut le cas au Whitney Museum de New York (Martel, 2006; Wu, 2002).

Les donateurs les plus généreux peuvent voir leur nom attribué à une salle ou à un bâtiment qu'ils ont contribué à rénover ou à créer ${ }^{22}$. Voir son nom associé à un espace d'exposition prestigieux est le couronnement d'une carrière philanthropique. Par leurs dons en argent ou en nature, ils permettent et accélèrent la réalisation de coûteux travaux, alimentant la frénésie de construction de musées. Alors que les extensions accroissent les budgets de fonctionnement, les dons ne les alimentent quasiment jamais (puisqu'ils ne seraient pas visibles).

\section{Le chantier permanent}

Beaucoup qualifient de fièvre muséale la création de (très) nombreux musées au cours des dernières décennies. De l'écomusée local aux gigantesques musées monumentaux, entre les rénovations, les agrandissements, les modernisations et les constructions, le paysage muséal mondial a effectivement beaucoup changé. En France, de 1982 à 1995, 120 nouveaux musées ont ouvert leurs portes (Krebs et Maresca, 2005). À Paris, entre les nouveaux musées (Orsay, Pompidou, quai Branly) et les transformations (Grand Louvre), l'offre culturelle a été décuplée en moins de 30 ans. Toujours plus grands, plus spectaculaires et plus onéreux, les musées en chantier semblent ne pas connaître de limites, ni géographiques ${ }^{23}$ ni financières, même si les coûts exorbitants de certaines réalisations commencent à susciter quelques questionnements ${ }^{24}$.

L'aspect le plus spectaculaire de cette fièvre muséale est l'usage immodéré de l'architecture monumentale. Certes, la qualité esthétique et architecturale des musées, espaces symboliques de représentation du pouvoir d'un mécène (qu'il s'agisse d'un individu ou de l'État), a toujours été l'objet d'une attention particulière. Mais, aujourd'hui, l'architecture tient lieu parfois de musée : le bâtiment serait un pis-aller de collection. L'exemple le plus connu est celui du Guggenheim Bilbao, dont l'architecture spectaculaire remplace quasiment la collection. En effet, malgré son nom (franchisé), l'essentiel des œuvres présentées n'appartient pas à la prestigieuse collection de la fondation Guggenheim, mais à une collection d'artistes locaux réunie par les autorités locales. Outre l'architecture du bâtiment, le nom de l'architecte lui-même devient symbolique, la signature devient icône. L'iconicité en architecture ne dépend pas uniquement des caractéristiques plastiques et esthétiques, mais surtout d'un système social de production de l'iconicité construisant les réputations et les hiérarchies entre les architectes, au-delà de leurs productions (Sklair, 2006). Un petit club très fermé d'architectes peut s'enorgueillir de monopoliser l'essentiel des marchés de maîtrise d'œuvre pour des musées et d'autres bâtiments symboliques ${ }^{25}$. C'est un jeu de réputation à double sens. Pour un architecte, apposer sa signature à un musée est un signe de reconnaissance et de prestige. Par essence, un musée est un espace de monstration de la création pour lequel l'architecte se doit de déployer son ingéniosité et sa créativité, sans contraintes (ou presque). Pour un musée, associer son nom à la signature d'un architecte prestigieux est un gage de légitimité et un outil de communication, car le bâtiment peut lui-même être l'objet d'une forte médiatisation. Par exemple, le Guggenheim Bilbao est utilisé dans de nombreuses publicités pour signifier l'inventivité et la modernité de produits aussi variés que les services bancaires ou les automobiles.

Une autre évolution spectaculaire est l'instrumentalisation de ces projets de musées dans des projets urbains. Le musée prend ainsi une nouvelle dimension : il devient le moteur d'une opération urbaine, le symbole de la transformation d'une ville et l'assurance de réussite d'un projet de reconversion postindustrielle. D'ailleurs, hier ouverts dans d'anciens palais, les musées sont aménagés aujourd'hui dans d'anciens bâtiments industriels, renforçant la patrimonialisation de ces espaces de la production manufacturière et du travail ouvrier, consacrant le passage à l'ère postindustrielle (Tate Modern, Istanbul Modern, etc.). Beaucoup de ces musées-spectacles s'inscrivent dans des projets urbains globaux visant à produire ce que Dennis Judd (1999) appelle des «bulles touristiques ", c'est-à-dire des périmètres urbains artificiels séparant l'espace du tourisme du reste de la ville. De la même manière qu'un parc à thème, ces bulles offrent de multiples expériences de loisirs et de divertissements dans un environnement paysagé, propre, attractif et sécurisé.

La production d'espaces consacrés au tourisme s'articulant autour d'un ou de plusieurs musées a marqué les villes dès le $\mathrm{XIX}$ e siècle. Les grandes expositions étaient l'occasion de créer de nouveaux quartiers dédiés à la consommation culturelle. À la même époque, des quartiers de musées ont été créés, comme l'île des musées de Berlin (Evans, 2001; Gold et Gold, 2005). Toutefois, aujourd'hui, cette production d'espaces urbains dédiés à la culture et à la consommation s'inscrit à l'intérieur de stratégies urbaines de développement économique. Ces sites, où s'accumulent les signatures architecturales iconiques, sont mis en scène par les villes comme des figures de proue du dynamisme urbain et comme symboles d'émergence de nouveaux centres urbains globalisés. Ainsi les Émirats arabes signifient leur importance économique par la création d'immeubles de bureaux de grande hauteur et révèlent leurs ambitions culturelles à travers les projets de quartiers culturels et touristiques comme l'île Saadiyat à Abu Dhabi. Ces enclaves de consommation culturelle et de loisirs sont considérées par de nombreux critiques comme les paravents glamour de la ville néolibérale (Davis et Monk, 2007). Or, la construction d'infrastructures culturelles ne garantit pas à elle seule la dynamisation culturelle d'un quartier et, souvent, la création artistique fuit ces secteurs culturels formatés et planifiés (Mommaas, 2004 ; Vivant, 2007a). Ailleurs, les projets de musées nomades du Tate Modern ou de la fondation Chanel intègrent le caractère éphémère du spectacle pour transformer le musée en événement urbain. 


\section{Illustration 1}

Plan de l'exposition Gilbert et Georges, Tate Modern (automne 2007, Londres). Au centre de l'exposition, une boutique et un bar.

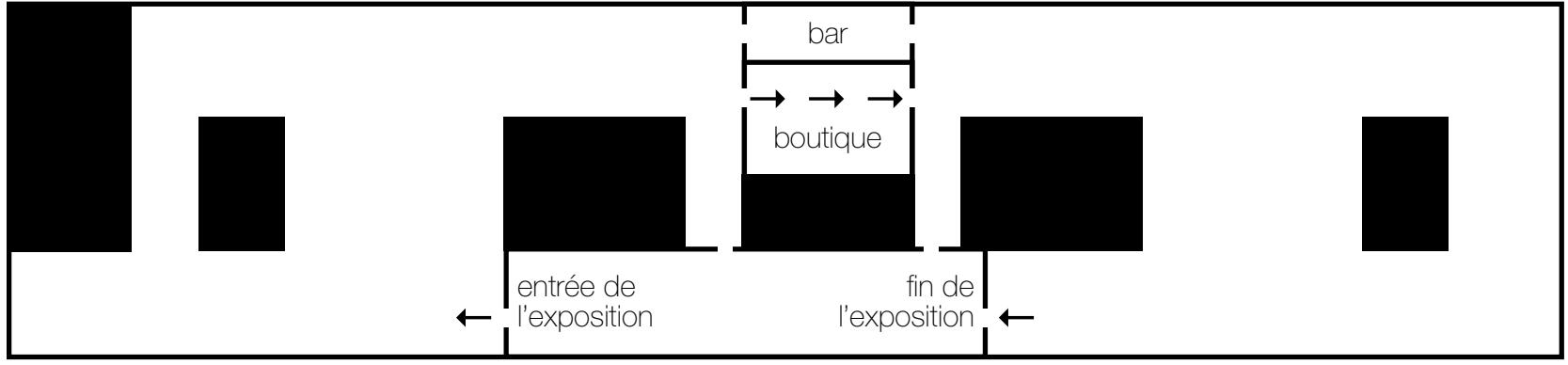

Source : Tate Modern ; graphisme : Elsa Vivant.

Par ailleurs, de nombreux musées ont engagé des travaux de restauration, d'extension ou de transformation, laissant toujours davantage de place aux espaces commerciaux (bars, restaurants, librairies, boutiques de souvenirs), permettant l'expansion d'activités génératrices de revenus importants pour le musée. Le mélange impromptu entre commerces et musées atteint son paroxysme au Carrousel du Louvre où un véritable centre commercial haut de gamme s'est glissé sous la pyramide ${ }^{26}$. Mais le commerce envahit aussi les espaces d'expositions eux-mêmes. Ainsi, lors de l'exposition Gilbert et Georges à la Tate Modern (droit d'entrée : 12 £), le visiteur devait traverser la librairie et la cafétéria au milieu de son parcours de visite (ill. 1).

De nombreux observateurs et critiques (comme Van Aalst et Boogaarts, 2002) interprètent le tournant actuel des musées comme leur transformation en Luna Park. En un sens, l'installation de Carsten Höller à la Tate Modern (dans le cadre des Unilever Series) en est le symbole et le révélateur. D'immenses toboggans installés dans le Turbine Hall ont été l'attraction de l'hiver londonien en 2007, attirant les foules et provoquant parfois de longues files d'attente avant qu'il soit possible au visiteur de se laisser glisser. Cette attraction divertissante est une représentation symbolique et critique de ce "glissement " des musées vers la marchandisation et la « détraditionnalisation » (certains diraient la désacralisation) de l'expérience muséale, ainsi que de son assimilation à un loisir comme un autre.

Le succès de quelques musées superstars s'explique par leur capacité à offrir au visiteur une expérience totale : à la fois lieu d'expositions, de concerts, de conférences, mais aussi de restauration, de détente, de rencontres et d'achats (Frey, 1998) ${ }^{27}$. De fait, outre l'émotion et le plaisir de la délectation suscités par les œuvres elles-mêmes, les motifs de visites d'un musée et de ses espaces commerciaux (restaurants, bars, librairies, boutiques de souvenirs) sont multiples : un déjeuner d'affaires sur le toit du Centre Pompidou, un rendez-vous galant sur les toboggans du Turbine Hall à la Tate Modern, une retouche de maquillage dans les toilettes d'accès public d'un Guggenheim, l'achat de cadeaux dans les boutiques du Carrousel du Louvre, voire une nuit en amoureux dans l'unique chambre de l'hôtel Everland sur le toit du Palais de Tokyo à Paris!

\section{La consommation de masse au musée : I'anti-marketing?}

Pour attirer toujours davantage de visiteurs, les musées développent des stratégies de communication de plus en plus poussées, produisant un discours narratif sur les collections et les choix artistiques, qui tendent, par le biais d'un design ou d'un logo, à transformer le musée en marque. Pour un musée, l'exploitation de sa "marque» consiste à produire du sens au-delà de la collection elle-même. II s'agit par exemple de susciter la visite pour des raisons autres qu'artistiques. Les commerces du Carrousel du Louvre proposent un "art du shopping». Le Victoria and Albert Museum a produit une campagne de publicité vantant son café, «le seul situé à coté d'un très beau musée». L'exploitation de la marque d'un musée passe entre autres par sa déclinaison en produits dérivés, comme les objets portant le logo du musée (stylo, badge, sac...) ou la reproduction d'œuvres des collections sous diverses formes (affiches, cartes postales, tee-shirts, livres d'art, bijoux...). Outre le renforcement de la réputation du musée, l'exploitation de sa marque par des produits dérivés ou des activités commerciales est une source de revenus ${ }^{28}$.

Les chiffres de fréquentation de certains musées sont vertigineux : notamment plus de 8 millions de visiteurs au Louvre en 2007. Toutefois, certains s'interrogent : perdu dans la foule, quel plaisir, quelle émotion peut désormais ressentir le visiteurconsommateur? Comment ressentir une communion esthétique avec l'artiste et son œuvre au milieu d'une foule bruyante? La course au gigantisme et au spectaculaire, la multiplication des espaces de consommation, l'attention portée aux statistiques de fréquentation comme unique mode d'évaluation mènent-elles les musées à leur propre perte? Du point de vue de certains 
spécialistes du marketing, il paraît contreproductif ou contre-tendance de rechercher sans cesse davantage de visiteurs, de bruits, de masse, alors que le calme et le caractère extraordinaire d'une expérience sont des qualités très porteuses sur certains marchés de niche (Guerzoni et Troilo, 2001). Ces critiques d'ordre artistique et esthétique prennent parfois une tonalité plus polémique et politique en mettant en cause la pertinence et les effets induits des politiques de démocratisation culturelle ${ }^{29}$

\section{De la marque à l'essaimage : la globalisation des musées}

La promotion et la communication des musées par la mise en œuvre de logique de marque muséale passe aujourd'hui par la création de branches de musées. L'ouverture de branches répond à une logique d'expansion, dont les conséquences sont pour l'instant inconnues. La fondation Guggenheim symbolise la marque muséale globale dont il convient de rappeler quelques faits méconnus relativisant son exemplarité. D'une part, l'initiative du musée Guggenheim Bilbao vient des autorités basques qui ont contacté la fondation pour qu'elle vienne installer une antenne dans leur ville en pleine restructuration. Peu enthousiasmé, son directeur leur a fait une offre qui, de son propre aveux, n'était pas très équilibrée (Krens, 2001), mais que les Basques ont acceptée : la fondation accordait une franchise pour 20 ans, d'un montant de 18 millions d'euros, pour la réalisation d'un bâtiment dont la fondation choisirait l'architecte, mais dont les coûts de construction (160 millions d'euros) et de maintenance seraient pris en charge par les autorités locales. En échange, la fondation s'engageait à fournir une expertise scientifique et artistique et à organiser régulièrement des expositions, dont la majeure partie des œuvres exposées n'appartiennent pas à la collection de la fondation, mais à une collection d'œuvres d'artistes locaux achetées, pour l'occasion, par les autorités locales. Le succès immédiat (en termes de fréquentation) et l'impact sur l'image de la ville font rêver bien d'autres édiles qui depuis cherchent à attirer la fondation chez eux. De nombreux projets ont été étudiés, mais aucun n'a vu le jour jusqu'à présent ${ }^{30}$. D'autre part, le succès «commercial » de la

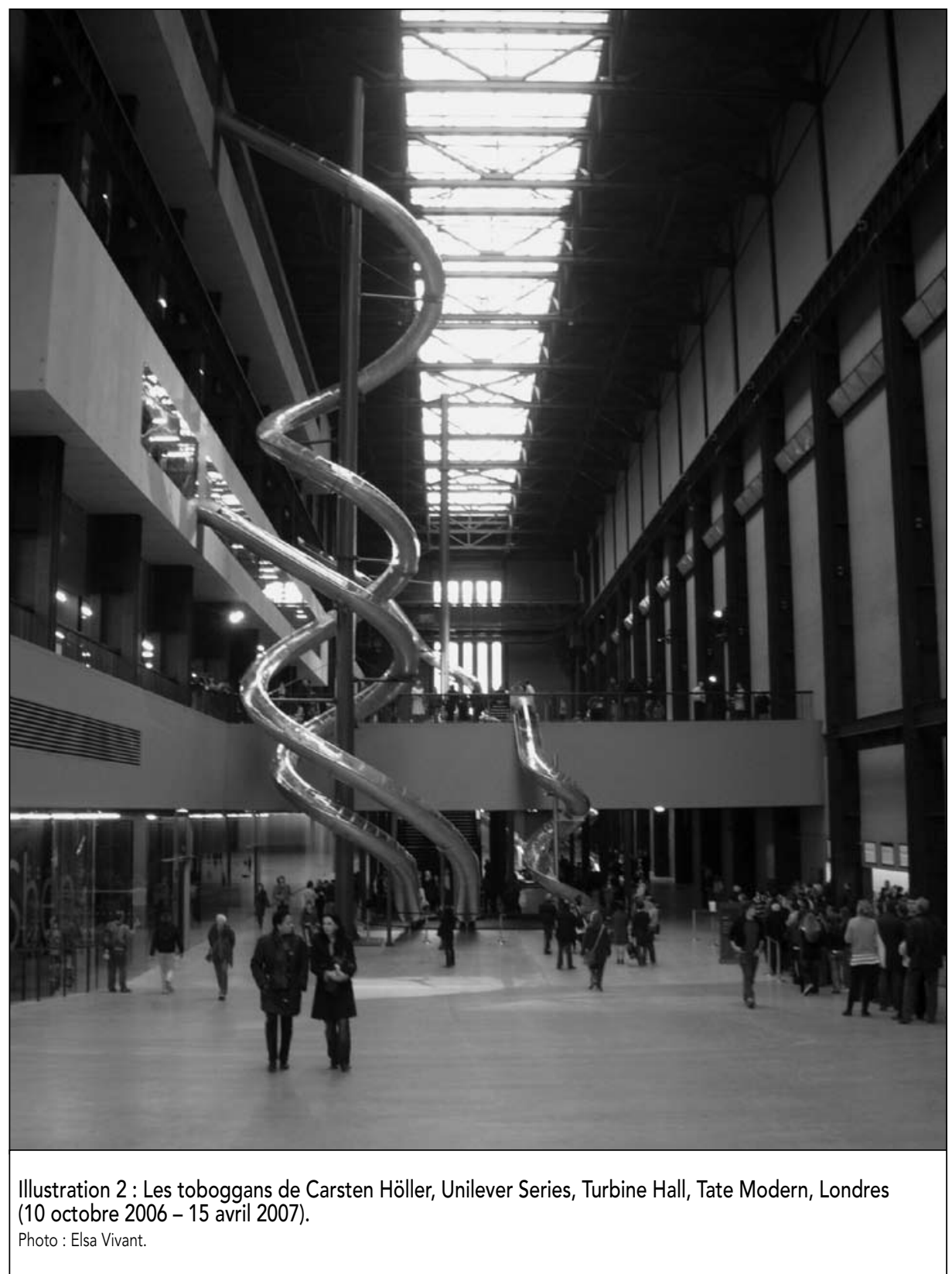

marque Guggenheim masque d'autres réalités moins glorieuses : des licenciements pour réduire la masse salariale, une pratique relativement courante de deaccessioning, la fermeture de la branche de Soho, des dérives commerciales dans les choix d'expositions très contestés... Le sobriquet "McGuggenheim " met en exergue les critiques portées sur ce musée (et son directeur, Thomas Krens), sur le truchement d'une collection par une architecture spectaculaire et sur la marchandisation de la culture et des musées qu'il symbolise (Baniotopoulou, 2001; Plaza, 2006; Vicario et Martinez Monje, 2003).
Les projets de création de branches des musées nationaux français s'inscrivent dans deux logiques différentes. Les branches du Louvre et du Centre Georges Pompidou en région (respectivement à Lens et Metz) poursuivent la décentralisation culturelle et la politique de démocratisation de l'accès à l'art en rendant accessibles les œuvres des musées parisiens aux habitants de province. Ces projets apparemment similaires s'inscrivent dans des contextes politiques, urbains et économiques différents. Le choix du site de Lens est le résultat d'une compétition entre six villes de la région, après l'annonce 
du Conseil régional du Nord-Pas de Calais d'accueillir et de financer une antenne du Louvre sur son territoire. Le Louvre-Lens est considéré comme un moteur de régénération urbaine d'une région sinistrée économiquement par la crise industrielle. Mais les impacts économiques et sociaux de ce musée devront être attentivement analysés après son ouverture, prévue en 2010, car il semble qu'ils soient quelque peu surestimés. Le fantasme projeté à partir de la success story du Guggenheim Bilbao éclipse les différences fondamentales entre les deux projets. En particulier, alors que le Guggenheim complétait un vaste projet urbain de régénération du port de Bilbao, le Louvre est en cours de construction à Lens alors qu'aucun projet urbain n'a encore été élaboré. Dans le cas du Centre Pompidou, même s'il s'inscrit dans un véritable projet urbain profitant de l'aubaine de l'ouverture d'une gare TGV (train à grande vitesse), le choix de la ville de Metz semble répondre à des motivations très politiques puisque, au moment de la prise de décision, le ministre de la Culture et de la Communication, ancien directeur du Centre Pompidou, était élu au Conseil Régional de Lorraine et énonçait publiquement ses velléités de candidature pour les élections municipales.

Le cas très controversé du projet Louvre Abu Dhabi, lui, s'inscrit à la fois dans la stratégie de "valorisation des actifs immatériels [sic] " de l'État ${ }^{31}$, où le musée est une marque dont on va vendre le droit d'exploitation de l'image pour une durée limitée, et dans la perspective de la politique économique et diplomatique de la France au Moyen-Orient (Martin, 2008; Vivant, 2007b). Par un accord entre les États français et émiratie, en louant la marque Louvre pour une durée de 30 ans, la France s'engage à fournir une expertise scientifique, à organiser des expositions à partir des collections de ses musées nationaux, à aider à la constitution d'une collection nationale (Donnedieu de Vabres, 2007 ; MCC, 2007). Cette marque sera apposée à un nouveau musée créé par Jean Nouvel, construit sur l'île de Saadiyat, rejoignant d'autres institutions muséales (dont une franchise Guggenheim) et participant à la production d'un nouvel espace de tourisme culturel dans cette île dédiée au tourisme et aux loisirs de luxe. Cet engagement de la France, qui percevra au total près de un milliard d'euros pour cette coopération culturelle, se comprend au regard de sa politique étrangère dans la région et des relations commerciales entre les deux pays, les Émirats arabes unis étant le principal partenaire de la France au Moyen-Orient, notamment par l'achat d'équipements militaires, aériens et de télécommunication. Du côté émiratie, le projet de Saadiyat s'inscrit dans une stratégie post-pétrole globale. En prévision de l'épuisement des stocks, et en vue de diversifier leur économie, les Émirats, depuis une quinzaine d'années, mènent différents projets visant à faire de cette partie du golfe un centre névralgique de l'économie globale (par la création de centres d'affaires), des transports (par la constitution de plaques tournantes aériennes en lien avec les stratégies des compagnies aériennes locales ${ }^{32}$ ), du tourisme (des projets pharaoniques comme le Dubaï hôtel ou les Palm Islands sont déjà devenus des icones du tourisme global) et de la culture et de l'éducation (par la création de branches d'universités internationales prestigieuses (dont la Sorbonne française) (Bellet, 2008; Davis et Monk, 2007; Herzberg, 2008).

\section{Conclusion}

Les tendances mises en évidence ici concernent une minorité de musées, mais ceux-ci accueillent la majorité des visiteurs $^{33}$. Si ces changements affectent l'ensemble du monde muséal, la plupart des institutions ne recourent pas aux formes les plus extrêmes de ces pratiques. L'éducation artistique, la démocratisation culturelle et les enjeux esthétiques demeurent au centre de l'activité des musées et nombre d'entre eux n'intègrent pas ces nouvelles logiques. Toutefois, mettre l'accent sur quelques exemples un peu caricaturaux a les vertus heuristiques de l'analyse par idéaux-types et permet de révéler le tournant entrepreneurial pris par les administrateurs de musée. Par ailleurs, d'autres lieux de monstration de l'art échappent aujourd'hui à la logique muséale en insistant sur une logique de rencontre et de mise en présence du public et de l'acte créatif privilégiant une esthétique relationnelle (Bourriaud, 2001) à une esthétique matérielle : par la visite de l'atelier d'un artiste, par les rencontres entre artiste et public pendant le processus de création, par la conceptualisation et la dématérialisation de l'œuvre... Ces lieux ont surgi le plus souvent en dehors de la sphère institutionnelle, ouvrant les portes à de nouvelles pratiques et à de nouvelles utopies, tant artistiques que sociales. Longtemps considérés comme marginaux, ces lieux, comme les squats d'artistes, inspirent aujourd'hui les institutions culturelles publiques pour concevoir de nouveaux espaces artistiques, comme le 104, nouveau lieu culturel parisien, qui a ouvert ses portes en 2008 (Vivant, 2006).

Elsa Vivant est maître de conférences en urbanisme à l'Institut Français d'Urbanisme.

\section{Notes}

1 Par exemple, les retombées économiques de l'activité touristique générées par les visiteurs des musées britanniques sont estimées à 1,5 milliard de livres sterling par an (Travers, 2006).

2 «Museums are the way we connect our past with our present and our future. Museums are centers of knowledge, with a clear-and growing-role as educators. They support the creative industries. They act as a powerful engine for regeneration, and are a primary reason why overseas visitors come to this country. Their impact on the economy, and the tangible effect they have on the imagination and spirit of the people who visit them, is enormous. " (DCMS, $2005: 6$.

3 La présentation des œuvres par le biais des expositions temporaires prend modèle sur les salons parisiens organisés au XIX siècle, hauts lieux de la vie culturelle et sociale de la nouvelle bourgeoisie, par exemple le Salon de la Rose-Croix en 1892 : exposition d'artistes parfois méconnus, visites guidées, communication et publicité dans les journaux, événements associés tels que des conférences ou des concerts (Poulot, 2005).

4 Un exemple parmi d'autres : d'octobre 2007 à février 2009, une exposition consacrée à l'œuvre de Louise Bourgeois va tourner entre la Tate Modern (Londres), le Centre Pompidou (Paris), le Musée Guggenheim (New York) et le LAMoCA (Los Angeles Museum of Contemporary Art).

5 Dans le même temps, l'objet lui-même des expositions change et ne se cantonne plus au domaine des beaux-arts. Les musées scientifiques proposent une approche ludique de la science et du savoir. Par exemple, la Cité des sciences de la Villette (Paris) a 
présenté récemment deux expositions originales et amusantes à destination des enfants et des préadolescents : I'une sur le corps humain, l'autre sur la sexualité (Crad'expo et le Zizi sexue/). Certaines expositions procèdent aussi de la reconnaissance des cultures populaires et des formes les plus commerciales de la culture de masse. L'exposition Kylie (présentant les tenues de scène de la chanteuse pop Kylie Minogue), initialement créée en Australie, a été présentée au prestigieux Victoria and Albert Museum de Londres avant de tourner dans les principaux musées du Royaume-Uni. De même, l'univers des films Star Wars a été le prétexte et le cadre d'une exposition scientifique qui a circulé de par le monde en 2006.

6 Parmi les 30 expositions les plus importantes des Galeries nationales du Grand Palais de 1980 à 2004, 12 étaient consacrées à des peintres impressionnistes, trois à Picasso et quatre à des civilisations anciennes (Poulot, 2005)

7 Le musée du Louvre et le High Museum d'Atlanta ont signé un partenariat pour la période 2006-2009, selon lequel le Louvre organisera des expositions d'œuvres de ses collections à Atlanta, ainsi que diverses activités de médiation culturelle. Mais cet accord prévoit également une contrepartie financière. Le terme "location" n'est pas évoqué, mais ces prêts seraient associés à "la participation de la communauté des mécènes de ce musée au financement de la rénovation des galeries des

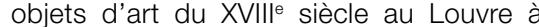
hauteur de 5346000" (Musée du Louvre, 2006 : 296).

8 Cette médiatisation est d'autant plus forte si des médias s'associent et sponsorisent l'exposition.

9 Site internet de l'Assemblée nationale, [http://www.assemblee-nationale.fr/13/ propositions/pion0233.asp], consulté le $1^{\text {er }}$ février 2008

10 Notons qu'un des rédacteurs du rapport, Jean-Pierre Jouyet, est secrétaire d'État aux affaires européennes depuis mai 2007.

11 Source : site internet de la présidence de la république française, [http://www.elysee.fr/elysee/elysee. $\mathrm{fr} / \mathrm{francais} /$ interventions/2007/juillet/ lettre_de_mission_du_president_de_la_ republique_adressee_a_mme_christine albanel_ministre_de_la_culture_et_de_la_ communication.79213.html], consulté le

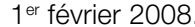

12 Ici le choix des mots n'est pas anodin. L'obscur deaccessioning, que l'on pourrait traduire par dessaisissement, est préféré à selling, plus évocateur.

13 L'Institut de France réunit l'Académie française, l'Académie des Inscriptions et Belles-Lettres, l'Académie des sciences, l'Académie des Beaux-Arts et l'Académie des Sciences morales et politiques. Cette institution publique est une des principales autorités morales et scientifiques en France.

14 Cette entreprise est une filiale du groupe Suez.
15 Le musée Jacquemart-André, la Villa Ephrusi de Rothschild, la Villa Kérylos.

16 Établissement public à caractère industriel et commercial sous tutelle du ministère de la Culture et de la Communication, qui regroupe les fonctions commerciales de plusieurs musées afin d'organiser et d'améliorer la recherche de ressources propres. La Rmn organise la plupart des expositions des musées nationaux (Benhamou, 2004).

17 Ainsi, l'établissement public du musée du Louvre regroupe le musée du Louvre, le musée Delacroix et les Tuileries.

18 Aux États-Unis, de nombreuses institutions à but non lucratif, dont des musées, possèdent un endowment, c'est-à-dire un capital placé en bourse, sous forme d'actions ou d'obligations, dont seuls les intérêts (non imposables) sont versés au budget de l'institution, le capital restant placé. La gestion de ce capital nécessite une expertise financière très pointue de même que la recherche de fonds nécessaires à l'alimenter et à l'augmenter. Ces tâches sont réalisées par un personnel très qualifié, au niveau de rémunération élevé, ce qui accroît d'autant les coûts de fonctionnement de l'institution (Martel, 2006). En France, la création d'un fonds de dotation pour le Musée du Louvre, alimenté par les contreparties financières du projet Louvre Abu Dhabi (400 millions d'euros), est actuellement à l'étude (Albanel, 2008).

19 Dans le cas des musées américains, les recettes se répartissent par tiers entre les subventions publiques, les ressources propres (billetterie, revenus du capital, produits dérivés) et l'apport des mécènes. En France, la part des subventions publiques tend à diminuer, même si environ les deux tiers des ressources des musées nationaux demeurent des financements publics (Benhamou, 2004).

20 II ne faut toutefois pas oublier que la majorité d'entre eux n'avaient aucun engagement philanthropique de ce type. Carnegie, Rockefeller, Ford et quelques autres demeurent des exceptions (Reich, 2008).

21 Le terme mode est pris à la fois au sens de tendance et de modalité.

22 Parmi de nombreux exemples, on citera le cas de l'aile Sainsbury de la National Gallery à Londres, construite grâce à un don exceptionnel des frères du même nom.

23 Les Émirats arabes rivalisent de méga-projets culturels (Herzberg, 2008).

24 Suite aux dépassements des coûts initialement prévus de plusieurs chantiers culturels, dont le très controversé et très cher (près de 300 millions d'euros) musée du quai Branly, la Cour des comptes (2007) a réalisé une enquête pour analyser les raisons du dépassement des budgets de la plupart des chantiers culturels et dégager des propositions pour le montage et la maîtrise d'ouvrage de ces projets.

25 Pour n'en citer que quelques-uns : Herzog et de Meuron (Tate Modern, Miami Art Museum), Sanaa (Louvre-Lens, MoCA New York), Franck Gehry (Fondation Louis Vuitton Paris, Guggenheim Bilbao et Abu
Dhabi), leoh Ming Pei (Grand Louvre, musée de Doha), Libeskind (musée juif de Berlin, Denver Art Museum), Jean Nouvel (Louvre Abu Dhabi, musée du quai Branly).

26 Les visiteurs payants du musée du Louvre dépensaient, en 2003, 25 euros en moyenne en achats divers, d'après une enquête réalisée par le musée et le CREDOC (Centre de recherche pour l'étude et l'observation des conditions de vie), citée dans Krebs et Maresca, 2005.

27 À ce propos, dans son livre très documenté (et très critique), Chin-Tao Wu (2002 : 138) cite l'ancien directeur de la Tate Modern : «I don't want the Tate to be a shopping mall. But if people want to buy something, they should be able to."

28 Par exemple, la location des espaces pour des événements spéciaux rapporte 309 millions d'euros au Louvre en 2006 (Musée du Louvre, 2006).

29 Un des conservateurs les plus importants du paysage muséal français, Jean Clair (2007 : 37), dresse un constat amer de l'expérience d'une visite de musée : "Le plaisir de visiter un musée a fini par succomber à la fatigue de son exercice : la queue, interminable, puis la cohue, les bousculades, le tumulte. Au lieu du paradis, un bruit d'enfer, l'assourdissement d'une salle des pas perdus ou de la verrière d'une piscine -, avec cet autre tourment de l'Enfer, la proximité des 'autres' : la promiscuité, la chaleur du coude-à-coude, l'impossibilité de demeurer immobile devant une œuvre sans voir se glisser dans son champ, incongrus, la nuque ou le bras d'un badaud, sans être distrait par les niaiseries échangées à propos d'un fessier de marbre opulent, sans être aveuglé par des éclairs de flash, et finalement sans être bousculé, poussé entraîné malgré soi dans un flux tantôt agité et tantôt languissant. »

30 Certes, la fondation possède, outre son siège newyorkais, d'autres branches : à Venise (collection de la nièce du fondateur), à Berlin (en partenariat avec la Deutsche Bank) et à Las Vegas. Mais aucun des projets sollicités par des municipalités (Taiwan Rio, Hong Kong, Salzbourg, Tokyo, Osaka, Guadalajara, etc.) et pour lesquels la fondation a facturé une étude de faisabilité n'ont pour l'instant abouti (Chu, 2007).

31 Sur la recommandation du rapport sur l'économie de l'immatériel (Lévy et Jouyet, 2006), une Agence du patrimoine immatériel de l'État a été créée au sein du ministère de l'Économie en avril 2007. Sa mission est de développer les conditions nécessaires à une "gestion dynamique» de ce patrimoine immatériel qui reste à recenser.

32 Dubaï ambitionne de créer d'ici 2010 le premier aéroport du monde. De manière générale, les projets urbains en cours dans la région semblent participer à une course à l'extravagance et à la dépense ostentatoire, comme l'idée de créée une ville nouvelle, Lyon Dubaï City, reprenant les éléments d'urbanité de la ville de Lyon et où seraient installées des branches des plus grandes institutions lyonnaises (sportive : olympique lyonnais; gastronomique : 
institut Bocuse; universitaire : Université Lyon 2; culturelle : musée des Soieries) (Landrin, 2008).

33 En France, quatre musées (musée du Louvre, musée du château de Versailles, musée d'Orsay et Musée national d'art moderne (Centre Pompidou) accueillent le tiers de l'ensemble des visiteurs de musées (DEPS, 2006).

\section{Bibliographie}

Abeles, Marc (2002), Les nouveaux riches. Un ethnologue dans la Silicon Valley, Paris, Odile Jacob.

Albanel, Christine (2008), Discours de Christine Albanel prononcé à l'occasion de la conférence de presse de présentation de Louvre 2020, projets et grands travaux, le 4 février 2008, Paris, Ministère de la Culture et de la Communication.

Azimi, Roxana (2003), "La gestion des arts confiée au privé", Le Journal des Arts, novembre.

Baniotopoulou, Evdoxia (2001), "Art for Whose Sake? Modern Art Museums and Their Role in Transforming Societies: The Case of the Guggenheim Bilbao ", Journal of Conservation and Museum Studies, vol. 1, $\mathrm{n}^{\circ}$ 7, p. 1-15.

Bellet, Harry (2008), « La bataille de l'art contemporain ", Le Monde, 6 février.

Benhamou, Françoise (2004), L'économie de la culture, Paris, La Découverte.

Bourriaud, Nicolas (2001), Esthétique relationnelle, Dijon, Presses du réel.

Chu, Chi-Jung (2007), «Re-examining Museums and Globalisation", Annual Congress of American Geographer, San Francisco, 12 avril.

Clair, Jean (2007), Malaise dans les musées, Paris, Flammarion.

Cour des comptes (2007), Rapport public thématique sur "les grands chantiers culturels ", Paris.

Davis, Mike et Daniel Bertrand Monk (dir.) (2007), Dreamworlds of Neoliberalism. Evil Paradises, New York, The New Press.

DCMS (Department for Culture, Media and Sport) (2005), Understanding the Future: Museums and the $21^{\text {st }}$ Century Life. The Value of Museums, London, Museums and Cultural Property Division.

DEPS (Département des études et de la prospective) (2006), "Les Musées de France en 2003 ", Les notes statistiques du DEPS, vol. $1, n^{\circ} 17$, Ministère de la Culture et de la Communication.

Donnedieu de Vabres, Renaud (2007), Allocution présentée lors d'une réunion avec les directeurs et les conservateurs des musées de France, le 16 janvier, Paris, Ministère de la Culture et de la Communication.

Evans, Graeme (2001), Cultural Planning. An Urban Renaissance?, London, Routledge.

Feldstein, Martin, (dir.) (1991), The Economics of Art Museums, Chicago, University of Chicago Press.
Frey, Bruno (1998), «Superstar Museums: An Economic Analysis", Journal of Cultural Economics, vol. 22, p. 113-125.

Gold, John et Margaret Gold (2005), Cities of Culture. Staging International Festivals and the Urban Agenda, 1851-2000, Aldershot, Aschgate.

Guerzoni, Guido et Gabriele Troilo (2001), «Pour et contre le marketing ", dans Jean Galard (dir.), L'avenir des musées, Paris, Éditions de la Réunion des musées nationaux, p. 135-154.

Harvey, David (1989), «From Managerialism to Entrepreneurialism: The Transformation in Urban Governance in Late Capitalism ", Geografiska Annaler. Series B, Human Geography, vol. 71, n 1, p. 3-17.

Herzberg, Nathaniel (2008), «Surenchère de méga-projets culturels", Le Monde, 6 février.

Judd, Dennis (1999), "Constructing the Tourist Bubble", dans Dennis Judd et Susan Fainstein (dir.), The Tourist City, New Haven, Yale University Press, p. 35-53.

Krebs, Anne et Bruno Maresca (dir.) (2005), Le renouveau des musées. Problèmes politiques et sociaux, Paris, La Documentation Française.

Krens, Thomas (2001), "L'attente des pouvoirs politiques ", dans Jean Galard (dir.), L'avenir des musées, Paris, Éditions de la Réunion des musées nationaux, p. 223-239.

Lai, Chia-Ling (2004), «Art Exhibitions Travel the World ", dans Mimi Sheller et John Urry (dir.), Tourim Mobilities. Places to Play, Places in Play, London, Routledge, p. 90-102.

Landrin, Sophie (2008), «Dubaï veut construire dans le sable une ville inspirée de Lyon ", Le Monde, 12 janvier.

Lévy, Maurice et Jean-Pierre Jouyet (2006), L'économie de l'immatériel. La croissance de demain, Rapport de la commission sur l'économie de l'immatériel, Paris, Ministère de l'Économie, des Finances et de l'Industrie.

Lindemann, Bernd Wolfgang (2001), «Financement public, mécénat et sponsoring ", dans Jean Galard (dir.), L'avenir des musées, Paris, Éditions de la Réunion des musées nationaux, p. 123-134.

Martel, Frédéric (2006), De la culture en Amérique, Paris, Gallimard.

Martin, Pauline (2008 [à paraître]), "La mondialisation des musées : variation d'un modèle du Guggenheim au Louvre ", dans Jérôme David et Thomas David (dir.), Les formes culturelles de la mondialisation (XIX ${ }^{-}$ $X X{ }^{e}$ siècles).

Mathur, Saloni (2005), "Museums and Globalisation ", Anthropological Quarterly, vol. 78, no 3, p. 697-707.

MCC (Ministère de la Culture et de la Communication) (2007), Création du musée universel Louvre Abou Dabi. Accord entre le Gouvernement de la République française et le Gouvernement des Émirats arabes unis, dossier de Presse.

Mommaas, Hans (2004), "Cultural Clusters and the Post-industrial City: Towards the
Remapping of Urban Cultural Policy ", Urban Studies, vol. 41, n 3, p. 507-532.

Moureau, Nathalie et Dominique SagotDuvauroux (2001), "Le Droit d'auteur confronté aux créations contemporaines", Mouvements, vol. 1, n 17, p. 17-20.

Musée du Louvre (2006), Rapport d'activité, Paris, Musée du Louvre.

Plaza, Beatriz (2006), «The Return on Investment of the Guggenheim Museum Bilbao", International Journal of Urban and Regional Research, vol. 30, n², p. 452-467.

Poulot, Dominique (2005), Musée et muséologie, Paris, La Découverte, coll. "Repères».

Rapetti, Rodolphe (2001), «L'expositionévénement", dans Jean Galard (dir.), L'avenir des musées, Paris, Éditions de la Réunion des musées nationaux, p. 55-67.

Reich, Robert (2008), Supercapitalisme. Le choc entre le système économique émergent et la démocratie, Paris, Vuibert.

Rigaud, Jacques (2008), Réflexion sur la possibilité pour les opérateurs publics d'aliéner des œuvres de leurs collections, Paris, Ministère de la Culture et de la Communication.

Schuster, Mark (1998), "Neither Public nor Private: The Hybridization of Museums", Journal of Cultural Economics, vol. 22, p. 127-150.

Sklair, Leslie (2006), "Iconic Architecture and Capitalist Globalization », City, vol. 10, no 1 , p. 21-47.

Travers, Tony (2006), Museums and Galleries in Britain. Economics, Social and Creative Impacts, Londres, London School of Economics and Political Science.

Van Aalst, Irina et Inez Boogaarts (2002), «From Museum to Mass Entertainment: The Evolution of the Role of Museums in Cities", European Urban and Regional Studies, vol. 9, n० 3, p. 195-209.

Vicario, Lorenzo et P. Manuel Martinez Monje (2003), "Another 'Guggenheim Effect'? The Generation of a Potentially Gentrifiable Neighbourhood in Bilbao", Urban Studies, vol. 40, no 12, p. 2383-2400.

Vivant, Elsa (2006), "Le rôle des pratiques culturelles off dans les dynamiques urbaines", thèse de doctorat, Institut Français d'Urbanisme, Université Paris 8, Champs-surMarne.

Vivant, Elsa (2007a), "L'instrumentalisation de la culture dans les politiques urbaines : un modèle d'action transposable? L'exemple de la création d'un quartier culturel à Newtown, Johannesburg (Afrique du Sud) ", Espaces et Sociétés, n 131, p. 49-66.

Vivant, Elsa (2007b), "Who Brands Who? The Role of Local Authorities in the Globalisation of Art Museums", Eura (European Urban Research Association) Annual Conference: The Vital City, Glasgow, 13 septembre.

Werner, Paul (2005), Museum, Inc.: Inside the Global Art World, Chicago, Prickly Paradigm Press.

Wu, Chin-Tao (2002), Privatising Culture. Corporate Art Intervention since the 1980s, London, Verso. 